\title{
Quantum-size effects in ultrathin Mg films
}

\author{
N. Binggeli ${ }^{1}$ and M. Altarelli ${ }^{2}$ \\ ${ }^{1}$ The Abdus Salam International Center for Theoretical Physics, Trieste 34014, Italy \\ and CNR-INFM DEMOCRITOS National Simulation Center, Trieste 34014, Italy \\ ${ }^{2}$ European XFEL Project Team, DESY, Notkestraße 85, 22607, Hamburg, Germany
}

(Received 3 August 2007; revised manuscript received 19 May 2008; published 23 July 2008)

\begin{abstract}
Recent experiments on the oxidation of ultrathin $\mathrm{Mg}$ films have revealed the existence of a correlation between surface reactivity and quantum-size effects. Using $a b$ initio density-functional calculations, we have investigated the electronic properties of epitaxial $\mathrm{Mg}(0001)$ films, 5-17 atomic-layer thick, on a $\mathrm{W}(110)$ substrate to clarify the origin of this correlation. We find that the decay length in vacuum of the thin-film local density of states at the Fermi energy exhibits a pronounced oscillatory behavior as a function of film thickness. This is expected to have a major impact on the electron transfer rate by resonant tunneling, which is believed to control the initial sticking of $\mathrm{O}_{2}$ molecules in the oxidation process. We have also examined the atomic-scale properties of the surface, interface, and quantum-well states of the $\operatorname{Mg}(0001)$ films on tungsten and the influence of epitaxial strain on the electronic states, in connection with the interpretation of recent photoemission spectra on these systems. In particular, we find strongly coupled surface-interface resonant states that originate from the Shockley surface states of the films. Comparison with photoemission measurements allows an unambiguous identification of the corresponding surface-interface-state splitting.
\end{abstract}

DOI: 10.1103/PhysRevB.78.035438 PACS number(s): 73.20.At, 73.21.Fg, 82.65.+r, 68.43.-h

\section{INTRODUCTION}

Quantum-well (QW) states in nanometer thick metal films have been associated with a number of interesting properties, such as magic layer numbers in thin-film growth, ${ }^{1-3}$ oscillatory magnetic interlayer coupling in magnetic multilayers, ${ }^{4-6}$ work-function modulations, ${ }^{7}$ and periodic anomalies in thinfilm conductance. ${ }^{8}$ Electron confinement in thin films can be achieved by an appropriate choice of a substrate which reflects electronic states. Fully localized QW states can be obtained, e.g., in the presence of an absolute energy band gap or of a symmetry-or wave-vector-dependent band gap of the substrate. QW states that couple, instead, to the Bloch states of the substrate produce resonances, whose degree of confinement in the film depends on the coupling. Direct observations of the energy levels or resonances induced by QW states are provided by photoemission spectroscopy. ${ }^{9}$ In particular, in the case of $\mathrm{Mg}(0001)$ films grown epitaxially on $\mathrm{W}(110)$, well resolved series of QW states and resonances have been reported in several recent photoemission studies. ${ }^{10-12}$

An exciting development related to QW states is the observation of a correlation between quantum-size effects and the surface reactivity of ultrathin $\mathrm{Mg}$ films. ${ }^{13}$ Experimentally, oscillations were observed in the oxidation rate of $\mathrm{Mg}(0001)$ films on W(110), as a function of film thickness, with the largest oxidation rate occurring when a $\mathrm{QW}$ state was found to cross the Fermi energy, $E_{F}$, in photoemission spectra taken near normal incidence. In particular, the changes observed in the initial oxidation rate-when most of the film was still metallic, were found to be dramatic. The precise mechanism behind such changes has been debated in the literature. Although the effect was initially suggested to be due to a change in the density of states (DOS) at, or near $E_{F},{ }^{13,14}$ the actual variations in the total DOS of the films at $E_{F}$ cannot simply account for the dramatic oscillations in the reactivity.
We recently proposed a theoretical interpretation of the experimental observations, in which the decay length in vacuum, $\lambda$, of the electronic local density of states around the Fermi energy was suggested to be the key parameter responsible for the changes in the reactivity. ${ }^{15}$ Modifications in $\lambda$ should have a direct, exponential influence on the electron transfer rate by resonant tunneling, which is believed to control the initial sticking of $\mathrm{O}_{2}$ on the metal surface. ${ }^{16}$ Here we present additional results, based on $a b$ initio calculations, on the electronic properties of $\operatorname{Mg}(0001)$ films in the range 5-17 atomic-layer thick on $\mathrm{W}(110)$, that provide further support to this interpretation. They confirm the presence of pronounced oscillations in $\lambda$, as a function of film thickness, with the periodic occurrence of maxima when a QW state with wave vector $k_{\|}=0$ (probed by normal-incidence photoemission) crosses $E_{F}$, consistent with the trend observed in the surface reactivity. We also examine the atomic-scale properties of the surface, interface, and QW states of the $\mathrm{Mg}(0001)$ films on tungsten and the influence of the substrate and of epitaxial strain on the electronic spectra. This allows us to clarify several unresolved issues concerning the interpretation of the photoemission spectra. The investigation of the influence of strain, in particular, provides a possible explanation for a systematic shift in the number of layers observed between calculated and experimental spectra. Based on the analysis of the electronic states with/without the substrate, we discuss the origin of the electronic states probed by normal-incidence photoemission and also address the evolution of these states along the $\overline{\Gamma S}$ direction of the $\mathrm{W}(110)$ surface Brillouin zone. Comparison with the experimental spectra yields an unambiguous identification of the two-band splitting associated with the formation of $\mathrm{Mg}(0001)$ Shockley surface-interface resonant states. The results are at variance with a recent interpretation of the surface states splitting in the photoemission spectra. ${ }^{12}$ 


\section{COMPUTATIONAL DETAILS}

The calculations were performed within densityfunctional theory, using the Perdew-Burke-Ernzerhof exchange-correlation functional. ${ }^{17} \mathrm{We}$ used scalar-relativistic Troullier-Martins pseudopotentials ${ }^{18}$ and a plane-wave basis set. The $\mathrm{Mg} 3 s, 3 p, 3 d$ orbitals and the $\mathrm{W} 5 d, 6 s, 6 p$ orbitals were treated as valence states, and we used the nonlinear core correction. ${ }^{19}$ The details on the pseudopotentials are given in Ref. 15. The epitaxial films were modeled using slab geometries in a supercell.

For thicknesses above 2 monolayers (ML), Mg films are known to grow epitaxially on $\mathrm{W}(110)$ with lattice parameters corresponding essentially to their bulk values. ${ }^{20}$ The mismatches between the experimental in-plane lattice parameters of $\operatorname{Mg}(0001)\left(a_{\mathrm{Mg}}=3.21 \AA, b_{\mathrm{Mg}}=\sqrt{3} a_{\mathrm{Mg}}\right)$ and $\mathrm{W}(110)$ $\left(a_{\mathrm{W}}=3.16 \AA, b_{\mathrm{W}}=\sqrt{2} a_{\mathrm{W}}\right)$ are $\sim 1.5 \%$ and $\sim 20 \%$ along the $\mathrm{W}[001]$ and $\mathrm{W}[1 \overline{1} 0]$ directions, respectively. ${ }^{10,20}$ Modeling such epitaxial systems, with the film and substrate each at their bulk lattice-parameters values, would require prohibitively large lateral dimensions of the supercell. We therefore elected to simulate unstrained $\mathrm{Mg}$ films on W(110), by laterally straining the $\mathrm{W}(110)$ slab to the in-plane lattice parameters of $\mathrm{Mg}(0001)$. The epitaxial alignment was made by positioning the atoms of the first $\mathrm{W}(110)$ layer, adjacent to the $\mathrm{Mg}$, in the continuation of the $\mathrm{Mg}(0001)$ hcp lattice. We used the calculated values of the bulk lattice parameters: $a_{\mathrm{Mg}}=3.19 \AA, c_{\mathrm{Mg}}=5.18 \AA$, and $a_{\mathrm{W}}=3.21 \AA$; relaxation of the $\mathrm{Mg}$ surface layers ${ }^{21}$ was found to have a negligible influence on $\lambda .{ }^{15}$ To assess the effect of the substrate, we also investigated the same $\operatorname{Mg}(0001)$ films isolated in vacuum (free-standing films).

For the Mg films on tungsten, we considered slabs including the $\mathrm{Mg}(0001)$ film on $7 \mathrm{ML}$ of $\mathrm{W}(110)$, terminated by 2 ML of Mg (0001). The Mg bilayer was introduced to avoid the presence of an electric field in the vacuum region separating the periodic images of the slab. We also used thicker slabs, with $13 \mathrm{ML}$ of $\mathrm{W}(110)$, to examine the spatial dispersion of the electronic states. We observed that the energy positions of the resonances and localized states corresponding to the surface, interface, and QW states of the Mg films changed by less than $0.05 \mathrm{eV}$ when going from 7 to $13 \mathrm{ML}$ of $\mathrm{W}(110)$. We used vacuum regions with a minimal thickness of $20 \AA$ to obtain well-converged values of $\lambda$. For the plane-wave expansion of the electronic states, we employed a kinetic-energy cutoff of $49 \mathrm{Ry}$ (14 Ry) for films with (without) the tungsten substrate. ${ }^{23}$ The self-consistent calculations were carried out using a $(20,20,1)$ Monkhorst-Pack $k$-point grid $^{24}$ and a Gaussian electronic-level smearing of 0.02 Ry to determine the Fermi energy. To evaluate the local density of states, we employed a $(48,48,1) k$-point grid centered at $\Gamma$ and a Gaussian smearing of $0.005 \mathrm{Ry}$. The partial density of states of the Mg films on tungsten was determined by integrating the planar average of the local density of states from a position at mid-distance between the interface $\mathrm{Mg}$ and $\mathrm{W}$ atomic layers to a position in the vacuum $10 \AA$ away from the outermost $\mathrm{Mg}$ atomic layer. The decay length $\lambda$ was obtained from a fit, assuming an exponential decay of the local density of states at distances beyond $\sim 2.15 \AA$ from the out-

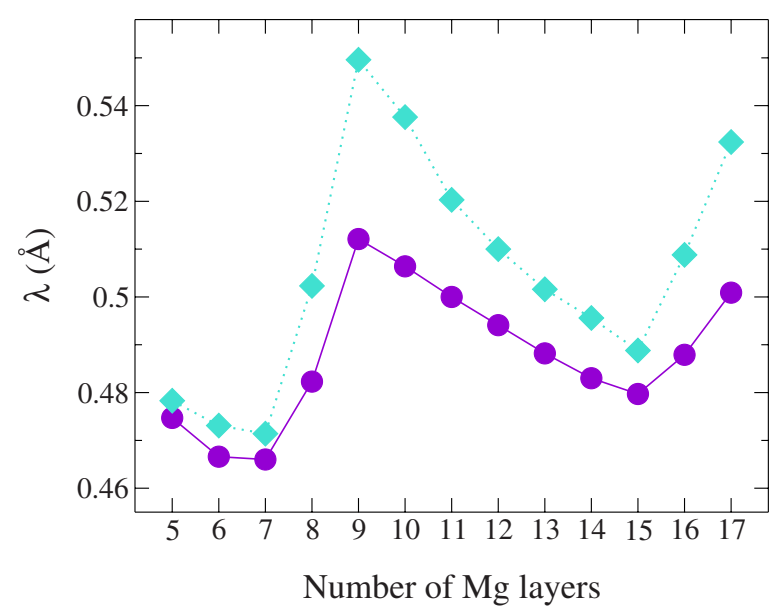

FIG. 1. (Color online) Calculated decay length in vacuum, $\lambda$, of the electronic local density of states at the Fermi energy of the $\mathrm{Mg}(0001)$ films on $\mathrm{W}(110)$ as a function of film thickness (disks). The decay length of the corresponding free-standing $\operatorname{Mg}(0001)$ films is also shown for comparison (diamonds).

ermost atomic plane. With the above parameters, the energy levels for a given slab were found to be converged to within $0.05 \mathrm{eV}$ and $\lambda$ to within $0.005 \AA .22$

\section{RESULTS AND DISCUSSION}

\section{A. Quantum-size effects on the decay length $\lambda$}

The calculated decay length $\lambda$ of the $\operatorname{Mg}(0001)$ films is displayed as a function of film thickness in Fig. 1, both for the $\mathrm{Mg}$ films on $\mathrm{W}(110)$ and for the free-standing $\mathrm{Mg}$ films. The behaviors are very similar in the two cases. The decay length shows pronounced oscillations, with a first minimum at 7 layers, a maximum at 9 layers, followed by a second local minimum at 15 layers, and an increase up to 17 layers. The presence of the tungsten substrate reduces the amplitude of the changes in $\lambda$ from $17 \%$ to $10 \%$, but has no significant impact on the position of the extrema. In Fig. 2, we show the partial densities of states at $\bar{\Gamma}$ of the Mg (0001) films on $\mathrm{W}(110)$ - which correspond to the states probed by normalincidence photoemission. The electronic levels at $\bar{\Gamma}$ of the free-standing films are also displayed in Fig. 2, for comparison. There is a close correspondence between the positions of the DOS peaks of the $\mathrm{Mg}$ films on $\mathrm{W}$ and the positions of the levels of the free-standing Mg films. The largest changes in the peak positions induced by the substrate is $\sim 0.2 \mathrm{eV}$ in the range $\left[E_{F}-8 \mathrm{eV}, E_{F}+2 \mathrm{eV}\right]$. The states indicated by "SS" in Fig. 2 originate from the $\operatorname{Mg}(0001)$ Shockley states of the isolated surfaces of the films; other features correspond to QW states of the films. The atomic-scale properties of these various states are presented in Sec. III B. With increasing film thickness an unoccupied QW states, in Fig. 2, is found to cross the Fermi energy at $\sim 9$ layers and a second one at 17 layers, which exactly coincides with the local maxima observed in the decay length $\lambda$ in Fig. 1.

The peak positions of the DOS, in Fig. 2, compare well with the near-normal-incidence photoemission measurements 


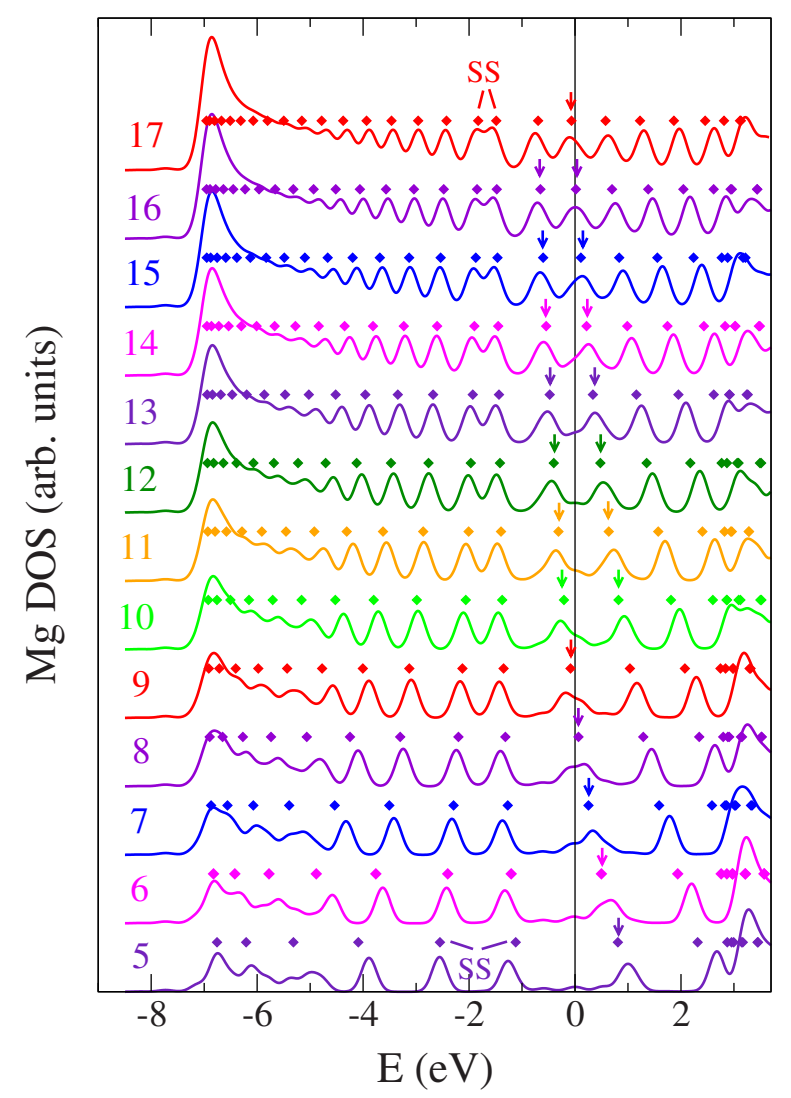

FIG. 2. (Color online) Calculated density of states at $\bar{\Gamma}$ of the $\mathrm{Mg}$ (0001) films on W(110) (solid lines). The thickness of the films ranges from 5 to 17 atomic layers (bottom to top curves). The densities of states have been convoluted with a Gaussian of width $0.2 \mathrm{eV}$. The symbols (diamonds) show the energies of the electronic levels at $\bar{\Gamma}$ of the free-standing $\operatorname{Mg}(0001)$ films. The states indicated by SS originate from the Shockley surface state of $\operatorname{Mg}(0001)$ (see text). The zero of energy corresponds to the Fermi level.

in the energy range $\left[E_{F}-4 \mathrm{eV}, E_{F}\right],{ }^{10,11,13}$ except for a systematic shift, by about $+2 \mathrm{Mg}$ ML, in the number of layers at which QW states cross the Fermi energy in the calculations relative to the experimental spectra. In the calculations, the first QW state with energy higher than the SS states enters the occupied-state spectrum at a Mg thickness of 8-9 ML, and the second one at a thickness of 16-17 ML. Experimentally, the corresponding QW states are found to enter the valence-band spectrum at 6-7 and $14-15 \mathrm{Mg} \mathrm{ML}{ }^{13}$ The same systematic shift is observed between the calculated maxima in $\lambda$ and the experimental maxima in the reactivity. ${ }^{13}$ We attribute this shift mainly to the presence of partially strained $\mathrm{Mg}$ layers near the interface. This will be discussed in Sec. III C, where we show that a lateral contraction of the $\mathrm{Mg}$ layers induces the entrance of a $\mathrm{QW}$ state in the valence spectrum at a lower number of Mg layers, as observed experimentally. We note that the calculated energy levels, in Fig. 2, compare relatively well (to within $\sim 0.2 \mathrm{eV}$ ) with the results of recent pseudopotential calculations performed for the free-standing $\operatorname{Mg}(0001)$ films. ${ }^{25}$

Our $a b$ initio results indicate that $\lambda$ has an oscillatory behavior with the number of $\mathrm{Mg}$ layers and is maximal when a QW state at $\bar{\Gamma}$ crosses the Fermi energy. ${ }^{26}$ Such a behavior can be explained based on a model description for $\lambda$ considering independent electrons in a square-well potential. ${ }^{15}$ The corresponding electronic states read:

$$
\psi_{n, k_{x}, k_{y}}^{E}(x, y, z) \sim \chi_{n}(z) e^{i\left(k_{x} x+k_{y} y\right)},
$$

and have energies $E=E_{n}+\hbar^{2}\left(k_{x}^{2}+k_{y}^{2}\right) / 2 m^{*}$, where $m^{*}$ is the electron effective mass and the $z$ axis is taken normal to the film. In the vacuum, $\chi_{n}(z)$ behaves as:

$$
\chi_{n}(z) \sim e^{-\alpha_{n} z},
$$

where

$$
\alpha_{n}=\left(\sqrt{2 m^{*}} / \hbar\right) \sqrt{-E_{n}},
$$

with the zero of energy taken at the vacuum level. $E_{n}$ coincides with the energy $E$ of the subband state $n$ at $k_{\|}=0$, measured relative to the vacuum level. For a given film thickness $L$, all states belonging to subband $n$, with energies $E \geq E_{n}$ have thus the same decay length $1 / \alpha_{n} \sim 1 / \sqrt{-E_{n}}$. Hence if $E_{F}$ is located between $E_{n}$ and $E_{n+1}$, the dominant decay length of the local density of states at $E_{F}$ is $1 / 2 \alpha_{n}$. With increasing width $L$ of the well, the energies of the QW states decrease with respect to $E_{F} ; \lambda$ thus first decreases as $1 / 2 \alpha_{n}$ $\sim 1 / \sqrt{-E_{n}}$, until the next QW state at $\bar{\Gamma}$ crosses $E_{F}$, at which point $\lambda$ increases to the new value $1 / 2 \alpha_{n+1} \sim 1 / \sqrt{-E_{n+1}} ; \lambda$ then decreases again with increasing $L$, displaying systematic oscillations with $L$. For a discrete number of atomic layers, the periodicity of the crossing of $E_{F}$ can be derived from the Bohr-Sommerfeld rule, which for $\operatorname{Mg}(0001)$ yields a periodicity of 7.7 ML. ${ }^{10,13}$ The particle-in-a-box model predicts thus oscillations in $\lambda$, with local maxima occurring when the highest-energy occupied QW state at $k_{\|}=0$ is closest to $E_{F}$, which accounts very well for the trends observed in Figs. 1 and 2.

The changes in the decay length reported here are expected to have a direct, exponential impact on the electron transfer rate by resonant tunneling-from the metal to the $\mathrm{O}_{2}$ molecule-which has been proposed to control the initial sticking of the oxygen molecules impinging on the surface, via the attractive image charge potential on the ionized $\mathrm{O}_{2}^{-}$ molecule. ${ }^{16}$ Assuming a transfer rate by tunneling proportional to $e^{-d / \lambda}$, with $d$ the distance between the metal surface and the center of mass of the molecule, and considering a typical distance $d$ of $3.5 \AA$-within the expected physisorption range of the $\mathrm{O}_{2}$ molecules, ${ }^{16,27}$ a $10 \%$ variation in $\lambda$ produces a $100 \%$ change in the transfer rate. Such a change is of the order of magnitude of the experimental change in the oxidation rate at low $\mathrm{O}_{2}$ exposure,,$^{13}$ and provides thus a very plausible explanation for the observed reactivity changes.

The oscillations in $\lambda$ that we predict from the $a b$ initio calculations are supported by a recent analysis ${ }^{28}$ of quantumsize effects in $\mathrm{He}$ scattering measurements for ultrathin $\mathrm{Pb}$ films on Ge. ${ }^{29}$ The latter changes were shown to be due mostly to a displacement of the surface electronic charge density, ${ }^{28}$ in contrast to previous interpretations invoking a displacement of the surface atomic layers. ${ }^{29}$ We note that the oscillations we find in the decay length of the local density of 


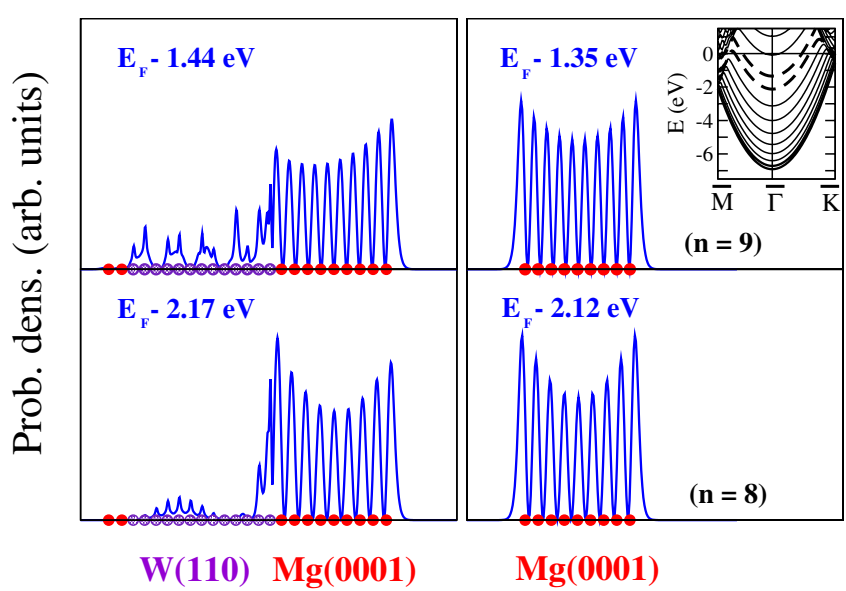

FIG. 3. (Color online) Left panels: Planar average of the probability density of the surface/interface states at $\bar{\Gamma}$, with energies $-1.44 \mathrm{eV}$ (a) and $-2.17 \mathrm{eV}$ (b), of the 9 monolayers $\mathrm{Mg}$ (0001) film on W(110). Right panels: Same as left panels, but for the corresponding states, with energies $-1.35 \mathrm{eV}$ (c) and $-2.12 \mathrm{eV}$ (d), of the free-standing film. These surface/interface states (labeled SS in Fig. 2) originate from the $\operatorname{Mg}(0001)$ Shockley states of the isolated surfaces of the film. The atomic-layer positions are indicated on the horizontal axis, at the bottom of each panel. The number of nodes, $n$, of the SS states of the free-standing film are shown in the bottom part of the panels. The inset shows the band structure of the 9-layer free-standing film, with the bands corresponding to the SS states indicated by dashed lines.

states near $E_{F}$ may also be related to a recent observation of quantum-size effects on the chemisorption properties of $\mathrm{Cu}(001)$ thin films ${ }^{30}$ Furthermore, KKR-layer calculations ${ }^{31}$ have indicated that the lifetime of negative ionic states of molecules, adsorbed on supported metal thin films varies with the thickness of the film, through coupling to QW states. This effect (not yet measured, to our knowledge) was associated, in the calculations, with oscillations in the amplitude, at the position of the molecule, of the density of empty states above $E_{F}$. This calculated behavior, consistent with the trend we find for $\lambda$, also support the interpretation we propose for the reactivity changes.

\section{B. SS, QW, and interface states}

In Fig. 3, we display the planar average of the probability density of the states label SS, in Fig. 2, for the 9-layer-thick $\mathrm{Mg}(0001)$ film. Both cases of the $\mathrm{Mg}$ film on tungsten and of the free-standing $\mathrm{Mg}$ film are considered. In the freestanding $\mathrm{Mg}(0001)$ film, the Shockley states of the two surfaces strongly overlap, and their interaction gives rise to a split pair of even- and odd-symmetry states relative to the midslab reflection (right-hand-side panels of Fig. 3). The splitting is as large as $0.8 \mathrm{eV}$ for the 9-layer film, and increases (decreases) to $1.4(0.4) \mathrm{eV}$ for the 5- (17-) layer film. In the presence of the $\mathrm{W}$ substrate, the SS states persist as strong surface/interface resonances, with maxima in the probability density located both in the region of the surface and of the interface $\mathrm{Mg}$ layer (left-hand-side panels of Fig. 3). The peaks of the SS resonances are only slightly shifted

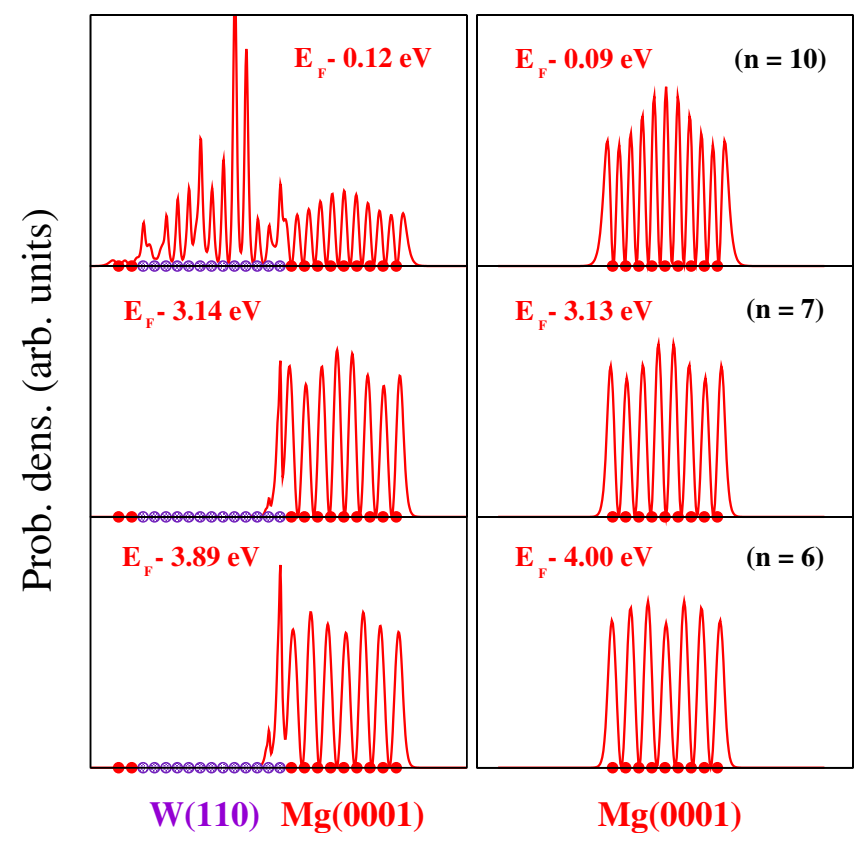

FIG. 4. (Color online) Planar average of the probability density of selected resonant/localized quantum-well states at $\bar{\Gamma}$ of the 9 monolayers $\mathrm{Mg}(0001)$ film on $\mathrm{W}(110)$ (left panels) and of the corresponding quantum-well states of the free-standing $\mathrm{Mg}(0001)$ film (right panels). The atomic-layer positions are indicated on the horizontal axis, at the bottom of each panel. The number of nodes, $n$, of the quantum-well states of the free-standing film are shown in the top part of the panels.

(by $0.15 \mathrm{eV}$ or less) relative to the corresponding levels of the free-standing films. The ratio of the probability density maxima at the surface relative to the interface is higher (lower) for the high- (low-) energy SS state, and increases (decreases) with increasing film thickness.

In Fig. 4, we show the planar average of the probability density of the QW states at $\bar{\Gamma}$ with energy in the range $\left[E_{F}\right.$ $\left.-4.5 \mathrm{eV}, E_{F}\right]$, for the supported and free-standing 9-layerthick $\mathrm{Mg}(0001)$ films. Unlike the SS states - which are characterized by a larger probability density at the surface/ interface than in the bulk parts, the QW states of the isolated films display their largest probability density inside the film. Inspection of Fig. 4 indicates that in the presence of the $\mathrm{W}$ substrate the QW states with energies in the range $\left[E_{F}\right.$ $\left.-4.8 \mathrm{eV}, E_{F}-2.3 \mathrm{eV}\right]$, which correspond to a gap at $\bar{\Gamma}$ in the W(110) projected bulk band structure, remain fully localized within the Mg films. This is analogous to the confined QW states found near the Fermi energy in previous $a b$ initio calculations for Ag films on Fe. ${ }^{35}$ The present $\mathrm{Mg}$ QW states, instead, with energy near the Fermi level-corresponding to the features crossing $E_{F}$ in Fig. 2-become broad resonances with a large probability density within the $\mathrm{W}$ substrate. The resonant character of the QW states which cross the Fermi energy tends to smoothen the oscillations in $\lambda$, in Fig. 1, relative to the case of the free-standing films.

Two angle-resolved photoemission studies have recently probed the layer-dependent electronic structure of $\mathrm{Mg}(0001)$ films on W(110) with similar experimental observations, but conflicting interpretations. ${ }^{11,12}$ The difference concerns the 
interpretation of the splittings of the $\operatorname{Mg}(0001)$ surface state. A splitting of a few tenths of an $\mathrm{eV}$ of the surface state was reported, for $\mathrm{Mg}$ thicknesses in the range 5-12 ML. ${ }^{11,12}$ This occurs in the $k_{\|}$-space region corresponding to a gap of the bulk $\mathrm{W}(110)$ projected band structure, in the energy range $\left[E_{F}-1.9 \mathrm{eV}, E_{F}\right]$, away from $\bar{\Gamma} \cdot{ }^{32,33}$ In Ref. 11 , this splitting was interpreted as a Rashba splitting of the $\operatorname{Mg}(0001)$ surface state, similar to the Rashba splitting previously observed for adsorbate-induced W(110) surface states. ${ }^{33,34}$ In Ref. 12, instead, the splitting (indicated by the solid lines in Fig. 4 of Ref. 12) was interpreted as a two-band splitting due to the parity-split Shockley states illustrated in Fig. 3. This interpretation was based on a highly qualitative comparison with $a b$ initio calculations for the split pair of Shockley states at $\bar{\Gamma}$ in free-standing Mg films (Fig. 3 of Ref. 12), which correspond to the SS states displayed in the present Fig. 3 (right-hand-side panels).

Although our calculations are scalar relativistic (spin-orbit averaged), and therefore cannot describe the Rashba splitting taking place at $k_{\|} \neq 0$, they are expected to describe well the Shockley surface-interface band splitting, especially at $\bar{\Gamma}$. In fact, in Sec. III A, we found good agreement between the calculated DOS of the films on tungsten and the experimental normal-incidence photoemission spectra. Our results also indicate that the tungsten substrate does not induce substantial changes in the energy positions of the SS states at $\bar{\Gamma}$, and cannot therefore account for a major quantitative difference between theory and experiment. Comparison of our results, in Fig. 2, with the measured photoemission data in Fig. 4(b) of Ref. 12 (for the $8 \mathrm{ML}$ film) indicates that, while the highest-energy surface state SS corresponds the " $\mathrm{S}$ " surface band with energy $\sim-1.5 \mathrm{eV}$ at $\bar{\Gamma}$ in Fig. 4(b) of Ref. 12, the lowest-energy SS state is about $0.9 \mathrm{eV}$ lower in energy and therefore corresponds to the band labeled "QW 1" in Fig. 4 of Ref. 12. The Shockley surface-interface-state splitting therefore cannot account for the other splitting observed in the $S$ band, in Ref. 12, which is $1 \mathrm{eV}$ higher in energy. Our results are therefore at variance with the interpretation proposed in Ref. 12.

In order to better understand the origin of the splitting observed in Refs. 11 and 12, we also performed calculations of the partial DOS of the $8 \mathrm{ML} \mathrm{Mg}$ film on tungsten at different $k$ points along the $\overline{\Gamma S}$ direction of the $\mathrm{W}(110)$ surface Brillouin zone (i.e., the direction also previously examined experimentally $\left.{ }^{11,33}\right)$. The corresponding band dispersion of the Mg-related states (the DOS of the Mg film) is shown in Fig. 5. In this figure we also display the edges of the valence gaps in the calculated $\mathrm{W}(110)$ bulk projected band structure (PBS) at the $k$ points considered. The main stomach gap of the $\mathrm{W}(110)$ bulk PBS-which is located, at $\bar{\Gamma}$, between -4.3 and $-2.3 \mathrm{eV}$ for strained $\mathrm{W}(110)$ — shrinks along the $\overline{\Gamma S}$ direction and eventually closes within the surface Brillouin zone. Going away from $\bar{\Gamma}$, the features associated with the main-stomach-gap states are seen to significantly broaden when crossing the gap edges, as expected from the interaction with the tungsten bulk states. Away from $\bar{\Gamma}$, another valence gap opens in the PBS at higher energy, for $k$ $\geq 0.25 \overline{\Gamma S}$, consistent with experiment. ${ }^{11,33}$ The most strik-

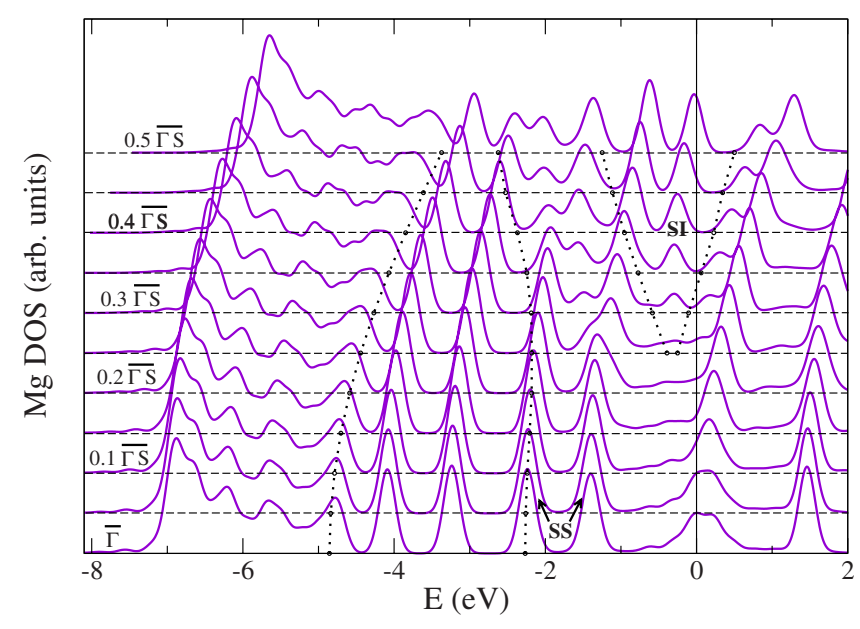

FIG. 5. (Color online) Calculated density of states of the $8 \mathrm{ML}$ $\mathrm{Mg}$ (0001) films on W(110) (solid lines) for different $k_{\|}$wave vectors along the $\overline{\Gamma S}$ direction of the W(110) surface Brillouin zone. The circles indicate the border of the gaps of the projected bulk band structure of W(110). The densities of states have been convoluted with a Gaussian of width $0.2 \mathrm{eV}$. The states SS originate from the Shockley surface states of the $\operatorname{Mg}(0001)$ film and "SI" labels the localized state deriving from the interacting W(110) surface state and $\mathrm{Mg}$ (0001) Shockley surface/interface states (see text). The zero of energy corresponds to the Fermi level.

ing feature concerning this gap is the occurrence of a new peak associated with a band of localized states within the gap, labeled "SI" in Fig. 5. We note that the highest energy SS state also leads to a second band of localized states at somewhat lower energy within that gap. This is in qualitative agreement with the experimental observations, ${ }^{11,12}$ although an additional smaller splitting was observed in Ref. 12.

In Fig. 6, we present the planar average of the probability density of the state SI and SS with energy $E_{F}-0.25 \mathrm{eV}$ and $E_{F}-0.81 \mathrm{eV}$, respectively, at the $k$ point $0.4 \overline{\Gamma S}$. The state SI has a dominant interface state character and originates

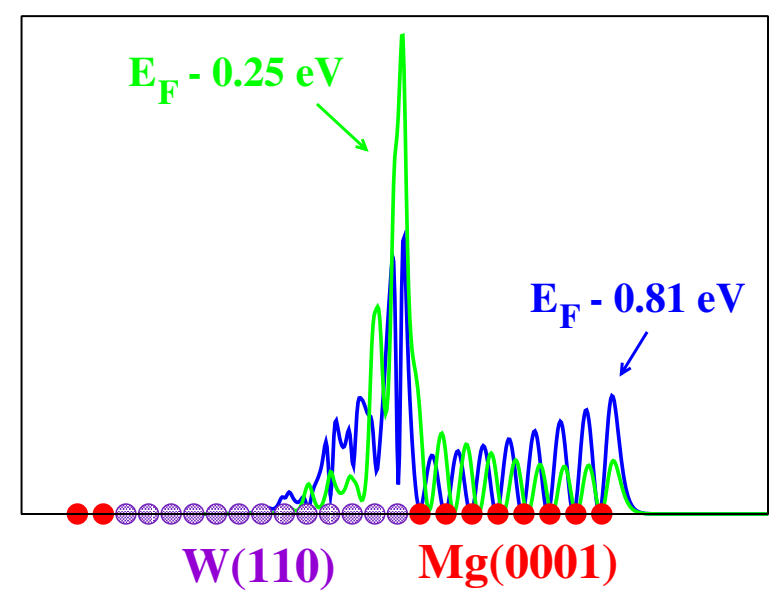

FIG. 6. (Color online) Planar average of the probability density of the surface/interface states of the $8 \mathrm{ML} \mathrm{Mg}(0001)$ film on $\mathrm{W}(110)$ with wave vector $k_{\|}=0.4 \overline{\Gamma S}$ and energies $E_{F}-0.81 \mathrm{eV}$ (SS state) and $E_{F}-0.25 \mathrm{eV}$ (SI state). The atomic-layer positions are indicated at the bottom of the figure. 
mainly from the interaction of the $\mathrm{W}(110) d$-derived surface state $^{33}$ with the $\operatorname{Mg}(0001)$ SS states. With increasing $k$ along $\overline{\Gamma S}$, the interface-state component tends to decrease somewhat and the SI state shows an increasing surface-state character. Although the "SS" related state with energy $E_{F}$ $-0.81 \mathrm{eV}$ has still a pronounced surface-state character, one can observe, in Fig. 6, that it also exhibits a strong interface state component, deriving from the $\mathrm{W}(110) d$ surface state. The latter contribution tends to increase somewhat with increasing $k$. The two-band states in the gap result from the interaction between the SS and W surface state. As we mentioned before, our calculations are scalar relativistic, therefore, some additional splitting (Rashba splitting) could occur, in principle, outside $\bar{\Gamma}$. Very recently, however, a comparative experimental study ${ }^{36}$ for $\mathrm{Mg}$ films deposited both on $\mathrm{W}(110)$ and on $\mathrm{Mo}(110)$ showed that the two-band splitting in the upper valence PBS gap is qualitatively similar for the two substrates, at different $\mathrm{Mg}$ coverage. As the spin-orbit interaction is negligible in the case of $\mathrm{Mo}^{33}$ this demonstrated that the Rashba effect is not the main origin of the observed splitting. The authors of Ref. 36 also pointed out that the parity splitting of the Shockley states is unlikely to be the origin of the observed splitting - although it could not be excluded. By comparing to photoemission data for the clean W(110) surface, they ascribed the latter splitting to the interaction of $\mathrm{Mg}$ states with $\mathrm{W}(110)$ surface-state resonances. Our results are consistent with this interpretation: we find that the parity-split Shockley states correspond to another pair of split bands and that the pair of localized states within the upper valence gap result from the interaction of the SS state with the W surface state.

\section{Effect of strain}

In order to understand the shift, between calculation and experiment, in the number of layers for QW states to cross the Fermi energy, we have investigated the effect of strain on free-standing $\mathrm{Mg}(0001)$ films. The strain due to the large lattice misfit between $\mathrm{Mg}$ and $\mathrm{W}$ is expected to be only partially released in the $\mathrm{Mg}$ layers closest to the interface. In fact, a recent low energy electron diffraction (LEED) study has indicated that the first $\mathrm{Mg}$ atomic layer, closest to the interface, is strongly contracted along the inplane $\mathrm{W}[1 \overline{1} \overline{0}]$ direction ( $b$ axis), relative to the bulk $\mathrm{Mg}(0001)$ lattice. ${ }^{20} \mathrm{~A}$ 9/10 (8/10) coincidence was observed between the first (second) $\mathrm{Mg}$ atomic layer closest to the interface and the $\mathrm{W}$ atomic layers, i.e., 9 (8) $\mathrm{Mg}$ atoms for every $10 \mathrm{~W}$ atoms, corresponding to a $\sim 11 \%(\sim 0 \%)$ contraction with respect to the bulk $\mathrm{Mg}$ lattice. Along the $\mathrm{W}[001]$ direction, the $\mathrm{Mg}-\mathrm{Mg}$ distance of the corresponding layers was found to coincide with the substrate lattice $(\sim 1.5 \%$ contraction relative to the bulk $\mathrm{Mg}$ ), within the experimental precision. In our calculations, we must restrict ourselves to consider identical lateral distortions in the different atomic layers of a given $\mathrm{Mg}$ film. Focusing thus on $\mathrm{Mg}$ films with thicknesses of 6-7 atomic layers - in the range where experimentally the first Fermilevel crossing takes place-an $11 \%$ contraction of the first $\mathrm{Mg}$ layer along the $b$ axis corresponds to an average lateral strain $\epsilon_{y y} \approx-0.02$ over these films.

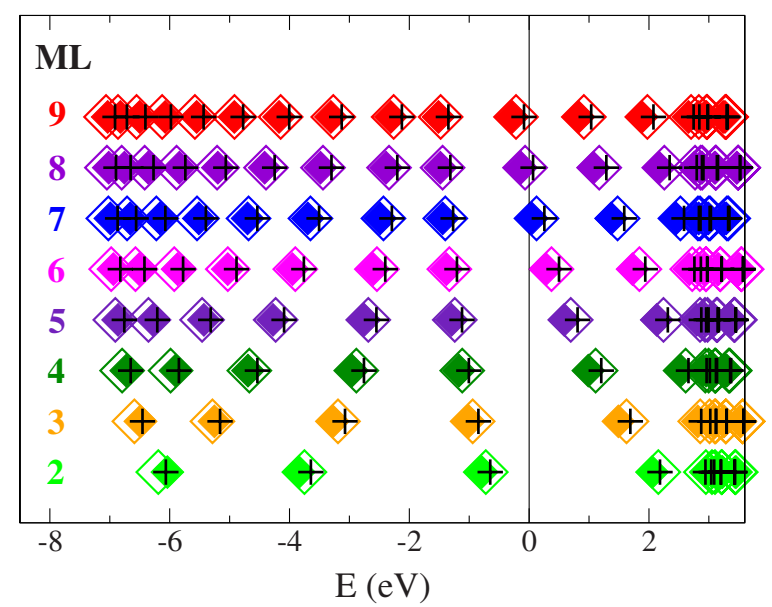

FIG. 7. (Color online) Calculated energy levels of free-standing $\operatorname{Mg}(0001)$ films to which a lateral contraction, $\epsilon_{x x}=-0.015$ and $\epsilon_{y y}=-0.02$, along the $\mathrm{Mg}[11 \overline{2} 0]$ and [10 $\left.\overline{10}\right]$ axis, respectively, has been applied. The solid symbols correspond to films which have been slightly elongated along the $\mathrm{Mg}[0001]$ axis, in response to the actual experimental inhomogeneous lateral contraction of the films (see text) and the empty symbols correspond to films whose length has been kept unchanged. The levels of the unstrained films are also shown for comparison (crosses). The films include 2-9 atomic layers (bottom to top spectra). The zero of energy corresponds to the Fermi level.

In Fig. 7, we display the electronic levels at $\bar{\Gamma}$ of freestanding $\operatorname{Mg}(0001)$ films to which we have applied a lateral compressive strain, $\epsilon_{x x}=-0.015, \epsilon_{y y}=-0.02$. Results are presented both for films which have been elongated in response to the actual inhomogeneous lateral contraction of the film, and films whose length has been kept unchanged. In the former case, the $\mathrm{Mg}-\mathrm{Mg}$ distance between the first two layers was increased by $5 \%$ - which corresponds to the calculated elongation of the free-standing bilayer for an average lateral strain $\epsilon_{x x}=-0.015$ and $\epsilon_{y y}=-0.055$, and the rest of the film was elongated by $0.5 \%$, as obtained from macroscopic elasticity theory for a strain $\epsilon_{x x}=-0.015 .{ }^{37}$ We also reported in Fig. 7, for comparison, the $\bar{\Gamma}$ levels of the unstrained films. The results indicate that the lateral compression alone induces the entrance of a QW state in the valence spectrum between 7 and $8 \mathrm{ML}$, compared to $9 \mathrm{ML}$ in the unstrained case. The elongation of the film then slightly enhances this trend, leading to the entrance of a QW state at about $7 \mathrm{ML}$. The reduction in the number of $\mathrm{Mg}$ layers for the entrance of a QW state produced by a lateral contraction of the Mg layers is consistent with the shift observed between calculations, for unstrained films, and experiment. The trends and estimate for the shift obtained here indicate that strain in the Mg layers near the interface is a possible explanation for the observed shift.

\section{SUMMARY AND CONCLUSIONS}

We have investigated, by means of $a b$ initio calculations, the electronic properties of epitaxial $\operatorname{Mg}(0001)$ films, with thicknesses between 5 and 17 atomic layers, on W(110). We 
find that the decay length in vacuum, $\lambda$, of the electronic local density of states at the Fermi energy exhibits pronounced oscillations with film thickness. The decay length is maximal when a quantum-well state passes through the Fermi energy. This behavior is explained based on particlein-a-box-model predictions for $\lambda$. The changes in $\lambda$ are expected to have a major impact on the tunneling rate in the electron transfer mechanism, which is believed to control the initial sticking of $\mathrm{O}_{2}$ in the oxidation process. We have also examined the atomic-scale properties of the surface, interface, and quantum-well states of the $\operatorname{Mg}(0001)$ films on tungsten and the influence of epitaxial strain on the electronic spectra, in connection with the interpretation of recent photoemission spectra on these systems. The investigation of the effect of strain, in particular, provides a possible explanation for the shift in the number of layers observed between calculated (unstrained) and experimental spectra. The study of the electronic states with/without the tungsten substrate show that the parity-split Shockley states of the isolated surfaces of the films persist as strong resonances in the presence of the substrate. Comparison with the photoemission spectra provides an unambiguous identification of the corresponding two-band splitting. Furthermore, the investigation of the electronic states of the film with wave vector $k_{\|}$along the $\overline{\Gamma S}$ direction of the surface Brillouin zone helps settling a controversy concerning the microscopic origin of two bands of $\mathrm{Mg}$ surface-related states located in a pocket gap of the $\mathrm{W}(110)$ projected bulk band structure.

\section{ACKNOWLEDGMENTS}

We thank L. Aballe, A. Barinov, M. Kiskinova, and A. Selloni for useful discussions. The computations have been performed using the PWscf package ${ }^{38}$ and carried out on the IBM sp5 computer at CINECA.
${ }^{1}$ A. R. Smith, K.-J. Chao, Q. Niu, and C. K. Shih, Science 273, 226 (1996).

${ }^{2}$ Z. Y. Zhang, Q. Niu, and C. K. Shih, Phys. Rev. Lett. 80, 5381 (1998).

${ }^{3}$ H. Hong, C.-M. Wei, M. Y. Chou, Z. Wu, L. Basile, H. Chen, M. Holt, and T.-C. Chiang, Phys. Rev. Lett. 90, 076104 (2003).

${ }^{4}$ S. S. P. Parkin, N. More, and K. P. Roche, Phys. Rev. Lett. 64, 2304 (1990).

${ }^{5}$ J. E. Ortega and F. J. Himpsel, Phys. Rev. Lett. 69, 844 (1992).

${ }^{6}$ R. K. Kawakami, E. Rotenberg, E. J. Escorcia-Aparicio, H. J. Choi, J. H. Wolfe, N. V. Smith, and Z. Q. Qiu, Phys. Rev. Lett. 82, 4098 (1999).

${ }^{7}$ J. J. Paggel, C. M. Wei, M. Y. Chou, D. A. Luh, T. Miller, and T. C. Chiang, Phys. Rev. B 66, 233403 (2002).

${ }^{8}$ M. Jalochowski and E. Bauer, Phys. Rev. B 38, 5272 (1988).

${ }^{9}$ T.-C. Chiang, Surf. Sci. Rep. 39, 181 (2000).

${ }^{10}$ F. Schiller, M. Heber, V. D. P. Servedio, and C. Laubschat, Phys. Rev. B 70, 125106 (2004).

${ }^{11}$ C. Koitzsch, C. Battaglia, F. Clerc, L. Despont, M. G. Garnier, and P. Aebi, Phys. Rev. Lett. 95, 126401 (2005).

${ }^{12}$ F. Schiller, R. Keyling, E. V. Chulkov, and J. E. Ortega, Phys. Rev. Lett. 95, 126402 (2005).

${ }^{13}$ L. Aballe, A. Barinov, A. Locatelli, S. Heun, and M. Kiskinova, Phys. Rev. Lett. 93, 196103 (2004).

${ }^{14}$ A. Hellman, Phys. Rev. B 72, 201403(R) (2005).

${ }^{15}$ N. Binggeli and M. Altarelli, Phys. Rev. Lett. 96, 036805 (2006).

${ }^{16}$ A. Hellman, B. Razaznejad, Y. Yourdshahyan, H. Ternow, I. Zoric, and B. I. Lundqvist, Surf. Sci. 532-535, 126 (2003).

${ }^{17}$ J. P. Perdew, K. Burke, and M. Ernzerhof, Phys. Rev. Lett. 77, 3865 (1996).

${ }^{18}$ N. Troullier and J. L. Martins, Phys. Rev. B 43, 1993 (1991).

${ }^{19}$ S. G. Louie, S. Froyen, and M. L. Cohen, Phys. Rev. B 26, 1738 (1982).

${ }^{20}$ L. Aballe, A. Barinov, A. Locatelli, T. O. Mentes, and M. Kiskinova, Phys. Rev. B 75, 115411 (2007).

${ }^{21}$ P. Staikov and T. S. Rahman, Phys. Rev. B 60, 15613 (1999).
${ }^{22}$ For $\mathrm{Mg}$ films on tungsten with thicknesses up to $6 \mathrm{ML}$ we also evaluated $\lambda$ and the partial DOS of the films using symmetric slabs (e.g., $6 \mathrm{Mg}$ ML/7 W ML/6 Mg ML), and compared the results to those obtained with the smaller asymmetric slabs terminated by the $\mathrm{Mg}$ bilayer. We found negligible changes (of at most $0.002 \AA$ ) in $\lambda$ and similarly the calculated partial DOS of the $\mathrm{Mg}$ film and the position of the Fermi energy within that DOS were found to be essentially undistinguishable in the symmetric and asymmetric slabs.

${ }^{23}$ C. J. Fall, N. Binggeli, and A. Baldereschi, Phys. Rev. B 65, 045401 (2001).

${ }^{24}$ H. J. Monkhorst and J. P. Pack, Phys. Rev. B 13, 5188 (1976).

${ }^{25}$ X.-G. Li, P. Zhang, and C. K. Chan, Physica B (Amsterdam) 390, 225 (2007).

${ }^{26}$ It should be noted that the calculated work function of the $\mathrm{Mg}$ films on W(110) also exhibits some changes with the number of $\mathrm{Mg}$ layers. However, these changes are very small: $0.05 \mathrm{eV}$ variation in the range 5 to $12 \mathrm{Mg}$ ML [consistent with previous calculations for unsupported $\mathrm{Mg}(0001)$ films, E. Wachowicz and A. Kiejna, J. Phys.: Condens. Matter 13, 10767 (2001)]. Furthermore, there is only an approximate correspondence between the minimum of the work function (at $8 \mathrm{ML}$ ) and the entrance in the valence spectrum of a new QW state at $\bar{\Gamma}$ (see also Ref. 7). All work function calculations were performed using symmetric slabs (see Ref. 15).

${ }^{27}$ C. Bungaro, C. Noguera, P. Ballone, and W. Kress, Phys. Rev. Lett. 79, 4433 (1997).

${ }^{28}$ L. Floreano, D. Cvetko, F. Bruno, G. Bavdek, A. Cossaro, R. Gotter, A. Verdini, and A. Morgante, Prog. Surf. Sci. 72, 135 (2003).

${ }^{29}$ A. Crottini, D. Cvetko, L. Floreano, R. Gotter, A. Morgante, and F. Tommasini, Phys. Rev. Lett. 79, 1527 (1997).

${ }^{30}$ A. G. Danese, F. G. Curti, and R. A. Bartynski, Phys. Rev. B 70, 165420 (2004).

${ }^{31}$ P. J. Rous, Phys. Rev. Lett. 83, 5086 (1999).

${ }^{32}$ K. W. Kwak, M. Y. Chou, and N. Troullier, Phys. Rev. B 53, 13734 (1996). 
${ }^{33}$ E. Rotenberg, J. W. Chung, and S. D. Kevan, Phys. Rev. Lett. 82, 4066 (1999).

${ }^{34}$ M. Hochstrasser, J. G. Tobin, E. Rotenberg, and S. D. Kevan, Phys. Rev. Lett. 89, 216802 (2002).

${ }^{35}$ C. M. Wei and M. Y. Chou, Phys. Rev. B 68, 125406 (2003).

${ }^{36}$ A. M. Shikin and O. Rader, Phys. Rev. B 76, 073407 (2007).

${ }^{37}$ The change was evaluated from macroscopic elasticity theory using the experimental values of the elastic constants, $c_{13}$ $=21.7 \mathrm{GPa}$ and $c_{33}=66.4 \mathrm{GPa}$, Elastic, Piezoelectric and related Constants of Crystals, Landolt-Börnstein, New Series, Group III, Vol. 11, edited by K.-H. Hellwege (Springer-Verlag, Berlin, 1979), pp. 42 and 179.

${ }^{38}$ S. Baroni, A. dal Corso, S. de Gironcoli, and P. Giannozzi (http://www.pwscf.org/). 